\title{
Image Restoration using a Network of Reduced and Regularized Neural Networks
}

\author{
Fillali Ferhat \\ Computer Science department, \\ University of Bordj Bouarreridj \\ 34000 BBA -ALGERIA-
}

\author{
Maza Sofiane \\ Computer Science department, \\ University of Bordj Bouarreridj \\ 34000 BBA -ALGERIA-
}

\author{
Graini Abid \\ Computer Science department, \\ University of Mohamed Boudief \\ 28000 Msila -ALGERIA-
}

\begin{abstract}
The aim of this paper is to implement an optimal neural network model to resolve the problem of colour image restoration which consists of retrieving original image degraded by invariant blur and corrupted by random white additive noise. We propose in this paper an algorithm which implements a general network of reduced neural networks model and adaptive regularization. The developed model is based on the original model of zhou and the modified model of Paik and katsaggelos.
\end{abstract}

The adaptive regularization parameter is used in our case when degradation model contains an additive component (additive noise) in order to obtain a compromise between image sharpness and noise elimination. It is chosen using an iterative algorithm which calculates the best value that maximizes the PSNR of the restored image.

Our model presents some improvements in terms of complexity and quality of restored images. It is shown by experiments that restored images obtained by the proposed model are better in terms of both numerical measurement and visual quality.

\section{General Terms}

Image restoration and reconstruction, neural network computing, regularization of ill posed problems.

\section{Keywords}

Image restoration, Artificial neural networks, filtering, debluring, Tikhonov regularization, optimization.

\section{INTRODUCTION}

Image Restoration is the process which attempts to obtain the original image from a degraded one, given all or partially knowledge of the degrading phenomenon. The occurring degradations include blur and noise. Blur can be caused when an object moves horizontally or vertically during an exposure or when the object is outside the camera's field, whereas noise is a random variation of brightness or colour information.

In the domain of image restoration, there are two categories of distortions: some of them are named spatially invariant where all pixels have suffered the same form of distortion, the relationship between the unknown and observed image can be described by a distortion model representing the PSF of the system $[1,2,3]$. Others are called spatially variant where the degradation suffered by a pixel depends upon its location in the image.

Image restoration is mostly considered as "ill-posed problem" i.e. a problem that does not have a unique solution, or the solution is not a continuous function of the data. Such problems are extremely sensitive to noisy data; that is, small perturbation of the data can lead to arbitrarily large perturbations in the solution. Many algorithms are used to approximate solution of ill-posed problem, such as Wiener Filter, Parametric estimation filter, Kalman Filter, algebraic methods using SVD or Constrained restoration [2, 3, 4].

Artificial neural networks (ANNs) are very general function approximators which can be trained based on a set of examples. Given their general nature, ANNs would seem useful tools for nonlinear image processing. Restoration of a high quality image from a degraded recording is a good application area of neural nets. Many restoration algorithms have been developed where the processing power lies in a large number of neurons (representing pixels) interconnected with synaptic weights.

In this paper, our main aim is to restore both gray level or colour images degraded by a known linear shift invariant distortion and random additive noise using an interconnected reduced and regularized neural networks. Our model is based on previous works (the original Hopfield model developed by Zhou et al in [5] and its modified version proposed in [6] by Paik and Katsaggelos which were implemented also in the same environment as the proposed algorithm using $\mathrm{C}++$ language, in order to compare them in term of efficiency). Our model was compared also with the technique of SVD and adaptive regularization (SVD_AR) presented by Fillali et al in [2].

This paper is organized as follows - In Section 2 we show the formulation of the problem of image degradation/restoration. Sections 3 and 4 describe the original Hopfield neural network model proposed by Zhou et al and its modified version developed by Paik and Katsaggelos. Section 5 describes some related works using other regularization techniques and neural network architectures. In section 6 , we present our proposition for image restoration problem using a general network of reduced neural networks combined with Tikhonov regularization technique. In Section 7 , we provide various experiments that demonstrate the effectiveness of the proposed model on different images degraded with different distortions. The obtained results are compared first with those of Zhou et al and Paik and Katsaggelos algorithms, and then we show its effectiveness relative to the SVD_AR technique. Section 8, we comment and discuss the obtained results. Finally, section 9 concludes the paper.

\section{PROBLEM FORMULATION}

In many practical situations, the image degradation can be modeled by linear blur (motion, defocusing ...) and the 
additive white Gaussian noise, then the degradation model is given by $[2,4]$ :

$$
g(i, j)=\sum_{x}^{N} \sum_{y}^{M} f(x, y) d(i, j, x, y)+n(i, j)
$$

Where $f(i, j), g(i, j)$ and $n(i, j)$ represent respectively, the original image, the corrupted image and the additive noise.

If $d(i, j)$ is a linear function then, $f(i, j), g(i, j)$ and $n(i, j)$ can be written as column vectors of size $N \times M$ and (1) is written as follows:

$g=D f+n$

$D$ is a matrix of size $N M \times N M$ where their elements are arrangement of $d(i, j)$ elements .

The main goal of image restoration process is to obtain an estimate of $f$ given $g$ and $D$ (without knowledge of additive noise variance).

In the domain of image restoration and reconstruction, a solution of (1) is obtained by minimizing a squared error plus a regularization term $[7,8]$ :

$$
E=\frac{1}{2}\left\|g-D f^{*}\right\|^{2}+\frac{1}{2} \lambda\left\|A f^{*}\right\|^{2}
$$

Where $f^{*}$ is the restored image, $A$ is a smoothness constraint operator and $\lambda$ is the regularisation parameter which mostly depends on the quantity of noise that exists in an image. The purpose to add the regularization term is to obtain a smoother solution when there is a noise. The value of $\lambda$ determines the balance of the needs for smoothness and fineness of reconstructed image.

i.e., find $f^{*}: \min \left\{\left\|g-D f^{*}\right\|_{2}^{2}+\lambda\left\|A f^{*}\right\|_{2}^{2}\right\}$

By defining energy function [7]:

$$
E\left(f^{*}\right)=-\frac{1}{2} f^{* t} W f^{*}+b^{t} f^{*}
$$

Where the matrix $W$ and the vector $b$ represent respectively the weight matrix and the bias vector of the Hopfield neural network model used for image restoration:

$$
\begin{aligned}
& W=-D^{t} D-\lambda A^{t} A \\
& b^{t}=-D^{t} g
\end{aligned}
$$

\section{ORIGINAL HOPFIELD MODEL OF IMAGE RESTORATION PROBLEM}

The original Hopfield neural network model (HNN) for image restoration was introduced by Zhou et al in [5]. Each neuron can take as a state two values: 0 and 1 . This structure is better suited for image processing in two gray levels: 0 for black and 1 for white. To restore a $r$ gray level image, each pixel should be represented by $r$ neurons.

In this case, the number of neurons in the HNN is $N \times M \times r$.
- The interconnection weights are updated by (6) as follows:

$w_{i, j}=-\sum_{p=1}^{M \times N} D_{p, i} D_{p, j}-\lambda \sum_{p=1}^{M \times N} A_{p, i} A_{p, j}$

- The bias is calculated using (6):

$b_{i}=\sum_{p=1}^{M \times N} g_{p} D_{p, i}$

The value of $\mathrm{j}^{\text {th }}$ pixel is given by:

$$
f_{j}^{*}=\sum_{k=0}^{r} s_{j, k}
$$

Where $S_{j, k}$ is the state of $k^{\text {th }}$ neuron of the $\mathrm{j}^{\text {th }}$ pixel.

- The input of each neuron is calculated by:

$$
x_{i}=\sum_{j=1}^{M \times N} w_{i, j} f_{j}^{*}+b_{i}
$$

The neuron's state is updated according to the following rule:

$$
S_{i}= \begin{cases}1 & \text { if } x_{i}>0 \\ 0 & \text { if } x_{i}=0 \\ -1 & \text { if } x_{i}<0\end{cases}
$$

The change in energy resulting from a change in neuron state $S_{i}$ is given by:

$\Delta E=-\frac{1}{2} w_{i i} S_{i}^{2}-x_{i} S_{i}$

If $\Delta E<0$, then the neuron's state is updated according to the following rule:

$$
s_{i, k}=s_{i, k}+S_{i} \text {. }
$$

The use of this model for image restoration requires a large amount of memory, due to the large number of neurons used and the large number of connections [3, 6]. In addition, the network consumes a huge amount of time to reach a steady state, due to the number of calculations for each neuron, during iterations.

\section{MODIFIED HOPFIELD NEURAL NETWORK MODEL}

To remedy the disadvantages mentioned earlier, J. Paik and A.K.Katsaggelos proposed in [6] a Modified Hopfield neural network model for solving the restoration problem which showed some improvements upon the algorithm proposed by Zhou. Their model manipulates complex structures of neurons; each pixel is represented by a single neuron which can take any value $\in[0$..max $]$ and is capable to update its state during each iteration.

The main difference over the algorithm of Zhou et al is the elimination of the step where the energy reduction is checked. They proposed to calculate $S_{i}$ as follows: 
$S_{i}= \begin{cases}1 & \text { if } x_{i}>\sigma_{i} \\ 0 & \text { if } x_{i}=\sigma_{i} \\ -1 & \text { if } x_{i}<\sigma_{i}\end{cases}$

Where:

$\sigma_{i}=-\frac{1}{2} w_{i, i}>0$

In this model, the number of neurons is reduced to $M \times N$ instead of $M \times N \times r$, while the number of connections is $M N \times M N$ instead of $M N r \times M N r$. This structure has the advantage of considerable reduction of the mass memory and the number of calculations.

The value of the $j^{\text {th }}$ neuron (pixel) is updated according to the following rule:

$f_{j}^{*}(t+1)=\varphi\left(f_{j}^{*}(t)+S_{i}\right)$

Where:

$\varphi(x)=\left\{\begin{array}{l}0 \text { if } x<0 \\ x \quad \text { if } 0 \leq x \leq \max \\ \max \text { if } x>\max \end{array}\right.$

This algorithm is much more efficient in terms of spatial complexity (the number of used neurons), and temporal complexity (faster than original algorithm).

\section{RELATED WORKS}

Several search models have been proposed for enhancement of the Hopfield neural network model of image restoration problem. Based on Paik and Katsaggelos model, Perry in [3] showed that the formula for the energy reduction resulting from a change in neuron state $S_{i}$ is a simple quadratic.

Then, it is possible to solve for the $S_{i}$ which produces the maximum energy reduction, by differentiating $\Delta E$, respecting to $S_{i}$ :

$\frac{\partial \Delta E}{\partial S_{i}}=-w_{i, i} S_{i}-u_{i}$

The value of $S_{i}$ which minimizes (12) is given by:

$-w_{i, i} S_{i}-u_{i}=0$

Therefore:

$S_{i}=\frac{-u_{i}}{w_{i, i}}$

In [9], Guan proposed a model-based Hopfield neural network evaluation and iterative gradient optimization and statistical filtering. He demonstrated that the iterative gradient method is efficient to implement the massive parallel processing structure of such neural network model.
All algorithms presented up to now consider gray level images. In the case of colour images, new techniques and other neural network models were developed. Zhang et al in [10] and Uma et al in [1] presented multilayer Morphological Neural network architectures for colour image restoration. Chema et al in [11] proposed a space variant neural network based on an autoregressive moving average (ARMA) process for blind image restoration (unknown PSF).

\section{PROPOSED MODEL}

By analyzing the original model of Zhou and the modified one of Paik and Katsagelos, we can observe that the matrix $D$ associated to the convolution mask $d(x, y)$ (which represents the degradation function) is constituted of $M \times N$ rows and $M \times N$ columns. The same observation can be made to the smoothness matrix $A$. Therefore, the weights matrix $W=D \times D-\lambda A A$ is of he same size as $D$. Although, few pixels within a certain neighbourhood of the current neuron will contribute to the neuron input calculation.

From these observations, we propose some optimizations on the calculation of the weights matrix and bias vector. The value of any neuron may be calculated by applying a certain mask applied on the current pixel. Therefore, we replace the weights matrix by a weights mask, and (6) and (7) can be replaced by (19) and (20):

$$
w(x, y)=d(x, y) \otimes d(x, y)-\lambda \Phi(x, y) \otimes \Phi(x, y)(19)
$$

Where the $\otimes$ operator denotes the convolution product. If $d$ is $l \times l$ mask, then $w$ will be a $(2 l-1)^{2}$ mask. $\Phi$ is also a $(2 l-1)^{2}$ mask that represents the smoothness constraint operator which is considered them in this paper as a Laplacian operator.

For the determination of the value of the regularization parameter $\lambda$, we have proposed to implement an iterative algorithm which attempts to obtain the highest value of $\lambda$ that maximizes PSNR of the restored image

The bias value of neuron $i$ is calculated using the following rule:

$$
b_{i}=\sum_{p=1}^{l} \sum_{q=1}^{l} d(p, q) \times g_{i}(p, q)
$$

Where $g_{i}$ is the $l$ neighbour matrix of the current pixel $i$.

For this reason, we propose to implement a network of reduced neural networks where the number of nodes equals the number of elements of the weights mask $w(x, y)$. Each reduced neural network will be responsible of the restoration of a single neuron.

Seen that each pixel contributes to the value of its neighbours, it is necessary to connect its restoration's network to all networks of its neighbour. Hence it is called a network of networks.

At any time $t$, we can process $M \times N$ single reduced and regularized networks formed of the current neuron (pixel) and its neighbours. This new structure can be schematized as follows: 


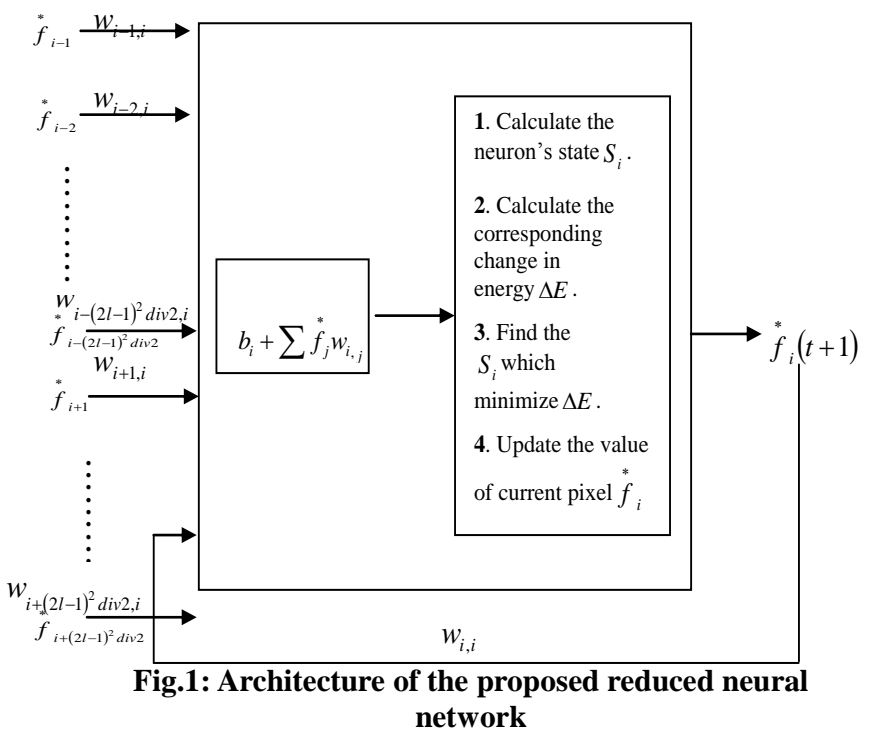

Where: $f_{i}^{*}, w_{i, j}$ and $b_{i}$ represent respectively the input, interconnection weight between neurons $i$ and $j$ and the bias. This new structure presents the advantage that it is possible to benefit of the parallel computing by distributing the execution of the algorithm across multiple processors, each processor will be responsible of the execution of a single restoration algorithm associated to a single reduced neural net.

If colour images to be restored are in RGB distribution, their restoration will be a difficult task because we must consider the correlation between the three colour subspaces. To remedy this problem, we propose to consider each subspace (red, green blue) as single gray level image space and are processed separately using the same neural network model. In this case, we implement three independents networks of reduced and regularized neural nets. Each network will restore a single subspace.

\section{EXPERIMENTATIONS}

We will present via this section the effectiveness of the proposed model on different images degraded by different distortions. Three objective parameters are calculated to show the quality of the restored image.

- The parameter $\triangle S N R$ indicates the improvement in the Signal to noise ratio. It is calculated as follows:

$$
\Delta S N R=10 \log _{10} \frac{\|g-f\|^{2}}{\|\hat{f}-f\|^{2}}
$$

- The parameter MSE (Mean Square Error) indicates the difference between original and restored images. It is calculated according to the following rule:

$$
M S E=\frac{\sum_{M, N}(\hat{F}(i, j)-F(i, j))^{2}}{M \times N}
$$

- The parameter $P S N R$ computes the Peak Signal to noise ratio. The higher is the PSNR the better the quality of the restored image. It is calculated as follows:

$$
P S N R=10 \log _{10}\left(\frac{255^{2}}{M S E}\right)
$$

Experiments 1, 2 and 3 show the effectiveness of the proposed model compared to the original model of Zhou et al and the modified model of Paik and Katsaggelos. While experiments

4, 5 and 6 shows its effectiveness relative to the SVD_AR algorithm.

\subsection{Experiment 1}

For the first experiment, a gray level image (the author) was degraded by a $5 \times 5$ low pass convolution filter modelling a Gaussian PSF. Figure 2.a shows the original image, while figures 2.b, 2.c, 2.d, 2.e show respectively the degraded image, the restored images using: Zhou algorithm, Paik and Katsaggelos algorithm and our proposed model.

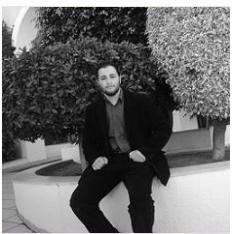

a. original image

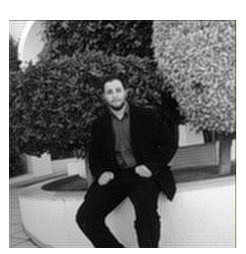

d. restored with

$\mathrm{P}$ and $\mathrm{K}$ algorithm $M S E=190.14$

$\triangle S N R=1.54$

$P S N R=2.34$

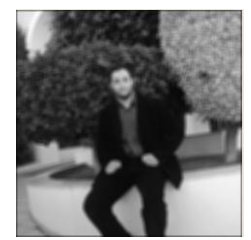

b. degraded image

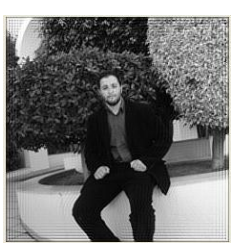

e. restored with

our algorithm

$M S E=56.94$

$\triangle S N R=5.14$

$P S N R=6.31$

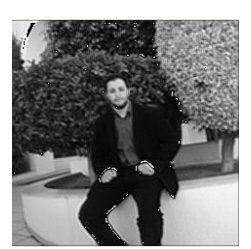

c. restored with Zhou algorithm

$M S E=195.35$ $\triangle S N R=1.45$

$P S N R=2.30$

Fig.2: Restoration of a burred image

\subsection{Experiment 2}

For the second experiment, a grey level image (a plane) was degraded by a $3 \times 3$ low pass convolution filter and corrupted by Gaussian additive white noise (variance=20). Figure 3.a shows the original image, while figures 3.b, 3.c, 3.d, 3.e show respectively the degraded image and the restored images using the three algorithms cited above.

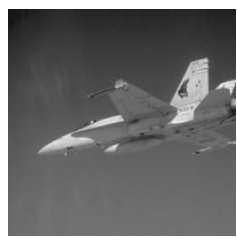

a. original image

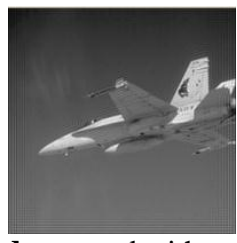

d. restored with

$\mathrm{P}$ and $\mathrm{K}$ algorithm

$$
\begin{gathered}
M S E=60.28 \\
\triangle S N R=0.14 \\
P S N R=4.19
\end{gathered}
$$

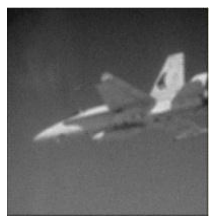

b. degraded image

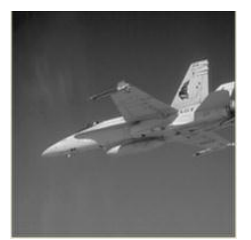

e. restored with our algorithm

$M S E=10.24$

$\triangle S N R=4.28$

$P S N R=24.90$

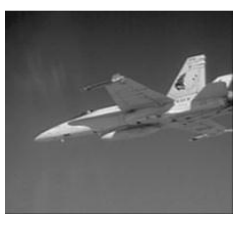

c. restored with Zhou algorithm

$M S E=23.15$

$\triangle S N R=3.22$

$P S N R=5.26$
Fig.3: Restoration of blurred and noisy image 


\subsection{Experiment 3}

For this experiment, we consider a colour image (author's brother) degraded by a $3 \times 3$ low pass convolution filter and corrupted by Gaussian additive white noise (variance $=15$ ). Because of there is no implementation of the algorithms of Zhou and Paik and Katsaggelos in case of colour images, we have implemented them by considering each RGB component as single gray level subspace. Figure 4 .a shows the original image, while figures 4.b, 4.c, 4.d and 4.e show respectively degraded and restored images using the three restoration models.

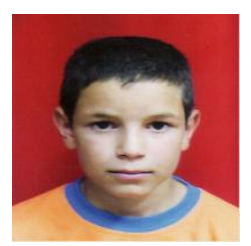

a. original image

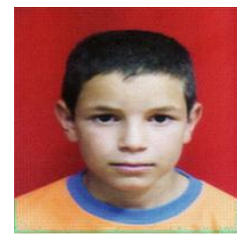

d. restored with

$\mathrm{P}$ and $\mathrm{K}$ algorithm

$$
\begin{aligned}
& M S E=22.55 \\
& \triangle S N R=0.59 \\
& P S N R=5.23
\end{aligned}
$$

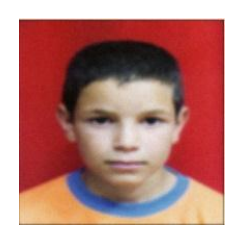

b. degraded image

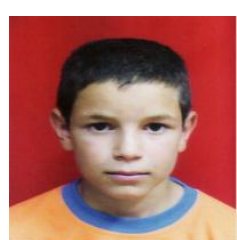

e. restored with proposed model

$$
\begin{gathered}
M S E=15.19 \\
\triangle S N R=2.26 \\
P S N R=8.99
\end{gathered}
$$

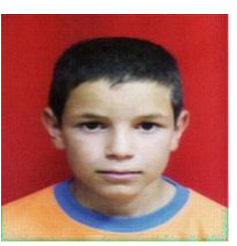

c. restored with Zhou algorithm

$M S E=21.59$

$\triangle S N R=0.98$

$P S N R=6.01$

Fig.4: Restoration of an RGB image degraded with Gaussian blur and corrupted by additive noise

\subsection{Experiment 4}

In this experiment, an MRI image was degraded by Gaussian blur of size $7 \times 7$. Figure 5 .a shows the original image, while figures 5.b, 5.c and 5.d show respectively the degraded image and the restored one using the neural model and SVD_AR technique.

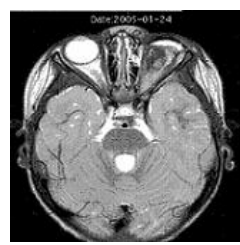

a. original image

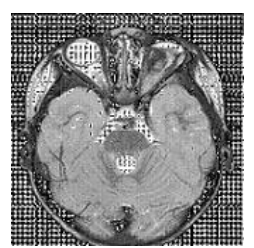

d. restored with

SVD_AR

$M S E=25.32$

$\triangle S N R=2.69$

Fig.5: Restoration of blurred MRI image using the neural model and the SVD_AR technique

\subsection{Experiment 5}

For this experiment, a 2 grey levels image was degraded by a Gaussian blur of size $9 \times 9$. Figure 6 .a shows the original image, while figures 6.b, 6.c and 6.d show respectively the degraded image and the restored one using the neural model and SVD_AR technique.

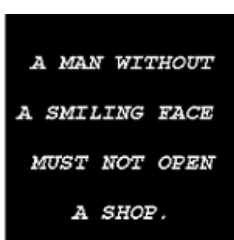

a. original image

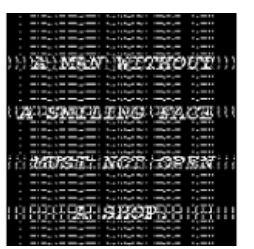

d. restored with

SVD_AR

$M S E=18.32$

$\triangle S N R=3.61$

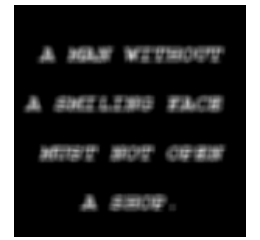

b. blurred image

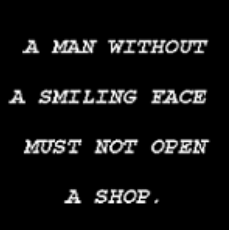

c. restored with our neural model

$M S E=1.39$

$\triangle S N R=23.71$

Fig.6: Restoration of 2 grey levels blurred image using the neural model and the SVD_AR technique

\subsection{Experiment 6}

The last experiment consists of the restoration of a noisy image (Saturn) which was degraded by important white additive noise (variance $=25$ ). Figure 7.a shows the original image, while figures 7.b, 7.c and 7.d show respectively the degraded image and the restored one using the neural model and SVD_AR technique.

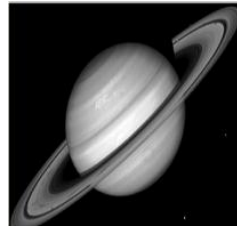

a. original image

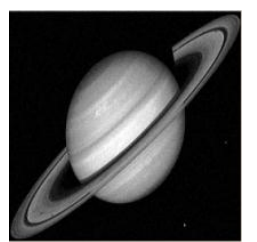

d. restored with

SVD_AR

$M S E=39.42$

$\triangle S N R=0.69$

Fig.7: Restoration of noisy image using the neural model and the SVD_AR technique

\section{PRACTICAL RESULTS DISCUSSION}

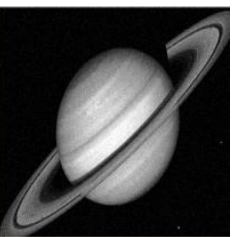

b. noisy image

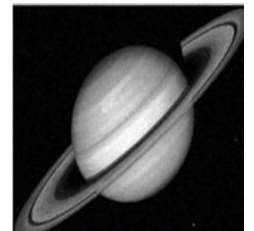
our neural model $M S E=14.93$ $\triangle S N R=4.01$ c. restored with

In this section, we discuss the effectiveness of the proposed model by analyzing the six experiments of the precedent section. On Figure 2, original image was blurred by a $5 \times 5$ 
low-pass convolution filter. The value of the regularization parameter $\lambda$ is zero because of the absence of the noise component in degradation model. In this case, the three algorithms quickly converge to the solution (we have considered just 40 iterations). The image is better restored using our model because it provided the best objective parameters values. Visually, there are little differences between the original image and the restored one.

On Figure 3, the original image was blurred using a $3 \times 3$ low-pass convolution filter. Gaussian additive noise was added after the blurring procedure (Figure 3.b). In presence of noise, the adaptive regularization leads to more and more iterations before to get close to the best solution. By choosing the regularization parameter $(\lambda=3)$ and stopping the execution after 100 iterations, it is shown that the proposed model provides better results (the highest value of SNR and PSNR).

On Figure 4, an RGB image was blurred by a $3 \times 3$ low-pass convolution filter and corrupted with Gaussian additive noise (Figure 4.b). As is mentioned in section 6, three independent networks were employed to restore both of the subspaces forming the image. The execution was stopped after 80 iterations, though the results are acceptable and the proposed model still providing the best results.

On figure 5 an MRI image was degraded by a $7 \times 7$ Gaussian blur. After 100 iterations, the proposed neural algorithm is more satisfying than the SVD_AR algorithm; restored image has better numerical measurements and visual quality.

On figure 6 , black and white image was degraded by a very important Gaussian blur. By stopping the execution after 60 iterations, the proposed neural algorithm dominates the SVD_AR algorithm; restored image with our neural model is very closer to original one.

Finally, on figure 7 noisy image was degraded by an important white additive noise (variance=25). After 100 iterations, the proposed neural model provides more satisfying result than the SVD_AR algorithm; restored image still has better numerical measurements and visual quality.

\section{CONCLUSION AND FUTURE WORK}

The approach of neural networks has been successfully applied in the domain of optimization such as image restoration. Different algorithms were proposed; they differ depending on the model of neural network and applied technique of regularization. In this paper, a general network of reduced and regularized neural networks is proposed to solve the problem of restoring noisy blurred images. The ideas presented are based on the works of Zhou, Paik and Katsaggelos and other researches in the domain of image restoration.

Experimentations results show that the proposed algorithm converges to images that are more accurate. The restored images have much better visual quality, higher improved SNR and PSNR and much lowest MSE. The proposed algorithm which is inspired from the two previous algorithms, gives also good results in terms of spatial and temporal complexity.

Comparisons were also made between the proposed algorithm and SVD technique presented by Fillali et al in [2]. We found that the proposed neural model provides better results than the SVD in cases of MRI images and the presence of additive noise in the degradation model; while the SVD has excellent results in the case of important blur and color images.

Currently our focuses are to improve the quality of restored color images by considering the correlation between the RGB components in the restoration model. For this, our future work will be based on construction of such model for video restoration. In fact, a video sequence is considered as a succession of still images. In this context, we will use our present model to achieve another one for video enhancement and restoration.

\section{ACKNOWLEDGEMENTS}

We want to express our deep and sincere gratitude to Pr. Khier Benmahammed, Professor at the Electronic department, University Ferhat Abbes - Algeria-, for his valuable suggestions, comments and constructive criticism which have indeed helped us to achieve this paper.

\section{REFERENCES}

[1] S. Uma, Dr. S. Annadurai, 2005. "Colour Image Restoration Using Morphological Neural Network", ICGST International Journal on Graphics, Vision and Image Processing, Volume 8 53-30.

[2] Ferhat Fillali, Khier Benmahammed and Graini Abid, 2010. "Image restoration using SVD and adaptive regularization" J. Automation \& Systems Engineering 43 173-181.

[3] Stuart William Perry, 1997. “Adaptive Image Restoration: Perception Based Neural Network Models and Algorithms", Ph.D. thesis in Electrical and Information Engineering, School of Electrical and Information Engineering, University of Sydney, NSW 2006.

[4] Jaroslaw Szost akowski, Andrzej Stajniak, 1996. "Neural Least - Squares Image Filter with Positive Constraints". Proceding of the International Workshop on Neural Networks for Identification, Control, Robotics, and Signal / Image Processing (NICROSP'96), Venice (Italy) 222-227.

[5] Zhou Y.T., Vaid A., Jenkins B.K., 1988. "Image restoration using a neural network", IEEE Trans. Acoust. Speech, Signal Processing, vol.36, 1141-1151.

[6] J.K. Paik and A.K. Katsaggelos, 1992. "Image Restoration Using a Modified Hopfield Network", IEEE Trans. Image Processing, vol. 1, no. 1, 49-63.

[7] Yi Sun, 2000. "Hopfield Neural Network Based Algorithms for Image Restoration and ReconstructionPart I: Algorithms and Simulations", IEEE Transactions on Signal Processing, Vol. 48, NO. 7, 2105-2118.1

[8] R. Youmaran and A. Adler, 2004. "Combining Regularization Frameworks for Image Deblurring: Optimization of Combined Hyper-parameters". In Proceeding of Canadian Conf. Electrical and Computer Engineering, Niagara Falls, Canada, 723-726.

[9] Ling Guan, "Model-based neural evaluation and iterative gradient optimization in image restoration and statistical filtering", Journal of Electronic Imaging 4(4), (1995) 407-412.

[10] Ling Zhang, Yun Zhang, Yi Min Yang, 2003. "Colour image restoration with multilayer Morphological Neural network", Proceedings of the second international conference on Machine learning and Cybernetics, Xian, 2831-2834.

[11] T. A. Cheema, I.M.Qureshi and A. Hussain, 2005. "Blind Image Deconvolution using a Space-variant Neural Network Approach", IEE Electronics Letters, Vol.41, No.6, 308-309. 